Chapter 10

\title{
Standard Electric and Magnetic Field for Calibration
}

\author{
Walter Gustavo Fano \\ Additional information is available at the end of the chapter
}

http://dx.doi.org/10.5772/intechopen.72687

\begin{abstract}
The electric and magnetic field from an electromagnetic wave usually are a matter of interest for regulatory standards to accomplish electromagnetic compatibility. Also in research works of technology in radio frequency, it is very important to analyze the electromagnetic fields. The electric and magnetic probes are studied and discussed in this chapter. The most important electric field probe is the dipole antenna, usually an electrically short dipole antenna. The loop antenna is used as magnetic field probe to measure the magnetic field. The current distribution and the electric circuit model for the dipole and the loop antennas are obtained. The antenna factor is also defined and calculated for the same antenna. This antenna factor allows to determine the incident electric field to the dipole antenna and the incident magnetic field to the loop antenna. The special cases of the electrically short dipole and the electrically short loop antennas are discussed. The Helmholtz coils are usually used to establish a known and uniform magnetic field zone for various applications. In the area where a uniform magnetic field is generated, sensor and magnetic field probe calibrations can be made in a low-frequency range.
\end{abstract}

Keywords: Maxwell equations, dipole antennas, loop antennas, electric field probe, magnetic field probe, Helmholtz coils, effective area, effective length, antenna factor

\section{Introduction}

This section explains the concepts that correspond to the maximum effective area and the effective length of a receiving antenna, when an electromagnetic wave is incident on the antenna. The concepts of electromagnetic waves, plane wave propagation in space, electric and magnetic fields, Poynting vector and power density incident on an antenna, dipole and loop antennas are required.

\subsection{Effective area and effective length}

In Figure 1a, an incident electromagnetic wave captures by receiving antenna, that is connected to a load impedance $Z_{L}$, can be observed. The electromagnetic wave is polarized in the $\mathrm{z}$ 


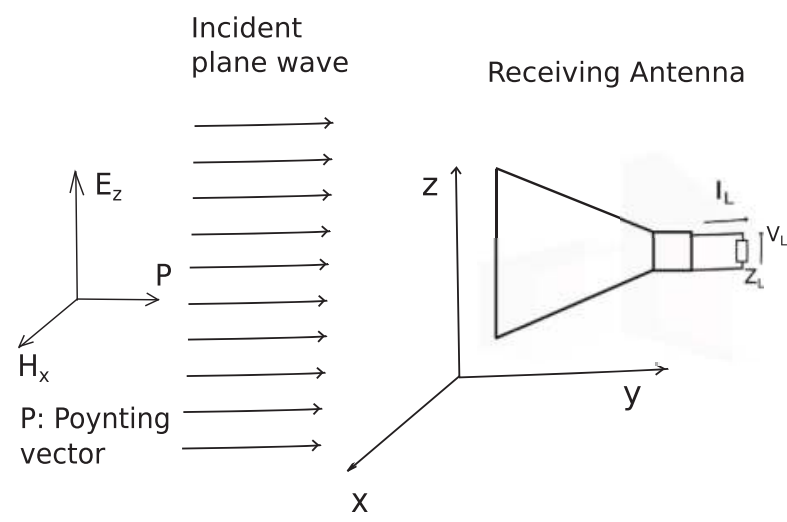

(a)
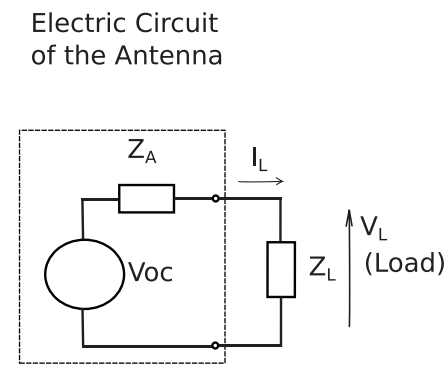

(b)

Figure 1. (a) Receiving antenna, with incident electromagnetic plane wave, parallel to the antenna. (b) the Thevenin equivalent electric circuit of the receiving antenna.

direction, which leads to a, and the antenna is oriented to the maximum gain direction. The incident power density of the electromagnetic wave [1] can be expressed in terms of the electric field as:

$$
P_{i}=\frac{\left|E_{i}\right|^{2}}{2 Z_{00}}
$$

where $Z_{00}$ is the intrinsic vacuum impedance, $P_{i}$ is the time average of incident power density, and $\left|E_{i}\right|$ is the intensity of the incident electric field.

The maximum effective area of an antenna can be defined as the ratio between the power received at the antenna terminals and the power density incident to the antenna, for linear polarization:

$$
A_{\text {eff }}=\frac{W_{R}}{P_{i}}
$$

In this case, the antenna is oriented to receive maximum power, and the load impedance $Z_{L}$ is matched to the impedance of the receiving antenna $Z_{A}$ :

$$
W_{R}=P_{i} A_{e f f}
$$

From Eqs. (1) and (3):

$$
W_{R}=\frac{\left|E_{i}\right|^{2}}{2 Z_{00}} A_{e f f}
$$

The equivalent electric circuit of a receiving antenna in Figure $\mathbf{1 b}$, where the incident electromagnetic wave is polarized in the $\mathrm{z}$ direction, and coincides with the polarization of the 
receiving antenna. The effective length of a receiving antenna can be defined as the ratio between the voltage induced at the terminals of the open circuit antenna and the incident electric field:

$$
L_{e f f}=\frac{\left|V_{o c}\right|}{\left|E_{i}\right|}
$$

where $\left|V_{o c}\right|$ is the voltage received at the terminals of the open circuit antenna and $\left|E_{i}\right|$ is the incident electric field.

The total apparent power in the load can be expressed thus:

$$
V_{L} \cdot I_{L}=\left|V_{L}\right|\left|I_{L}\right| \cos \phi+\left|V_{L}\right|\left|I_{L}\right| \sin \phi
$$

where $V_{L}$ is the voltage at the load, $I_{L}$ is the current flowing through the load, and $\phi$ is the phase difference between $V_{L}$ and $I_{L}$.

The active received power at the antenna terminals is:

$$
W_{R}=\frac{\left|V_{L}\right|^{2} \cos \phi}{Z_{L}}
$$

The relationship between the load voltage and the open circuit voltage is:

$$
\left|V_{L}\right|=\left|V_{o c}\right| \frac{\left|Z_{L}\right|}{\left|Z_{L}+Z_{A}\right|}
$$

From Eqs. (7), (8) and (5):

$$
W_{R}=\frac{\left|E_{i} L_{e f f}\right|^{2}\left|Z_{L}\right| \cos \phi}{\left|Z_{L}+Z_{A}\right|^{2}}
$$

From Eqs. (4) and (9):

$$
L_{\text {eff }}=\sqrt{\frac{\left|Z_{L}+Z_{A}\right|^{2} A_{\text {eff }}}{\left|Z_{L}\right| 2 Z_{00} \cos \phi}}
$$

This effective length is a general expression for any antenna rather than what is usually defined in the bibliography for dipole antennas [1].

\section{Electric field probes}

Consider a linear dipole antenna with an incident electromagnetic plane wave, polarized parallel to the antenna, which is captured by the antenna. The electric circuit of the antenna without load is shown in Figure 2. The voltage induced in the antenna terminals is denoted by $V_{o c}$. 


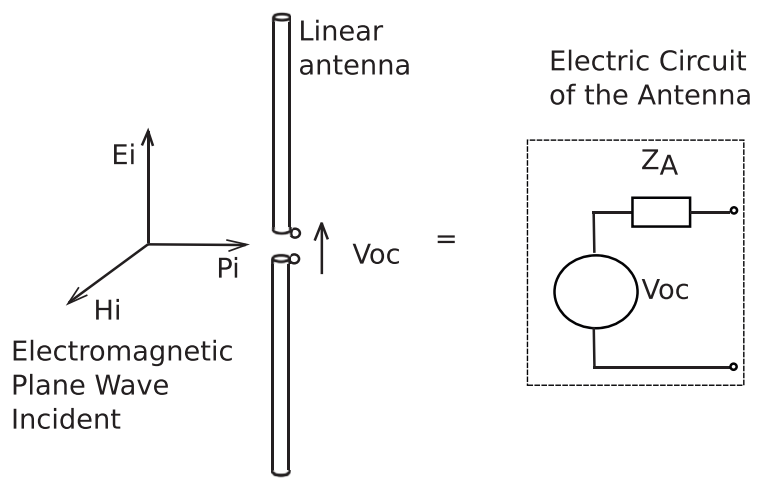

Figure 2. Receiving antenna, with incident electromagnetic plane wave, parallel to the antenna.

The effective length of a linear dipole antenna in receiving mode can be defined as a relation of electric field strength incident to the antenna and the voltage on the antenna, as shown in Figure 3, [2]. This can be expressed as:

$$
\left|V_{o c}\right|=\left|E_{i}\right| L_{e f f}
$$

where $\left|V_{o c}\right|$ is the amplitude of the open circuit voltage on the antenna, $\left|E_{i}\right|$ is the amplitude of the incident electric field parallel to the antenna, and $L_{\text {eff }}$ is the effective length of the linear antenna.

The received voltage is usually measured by the spectrum analyzer, which has the input impedance $Z_{L}=50 \Omega$, as shown in Figure 4. This receiving voltage and the receiving power can be expressed as:

$$
\left|V_{r}\right|=\sqrt{W_{r}\left|Z_{L}\right|}
$$

where $W_{r}$ is the power received by the spectrum analyzer and $Z_{L}$ is the impedance of the spectrum analyzer.

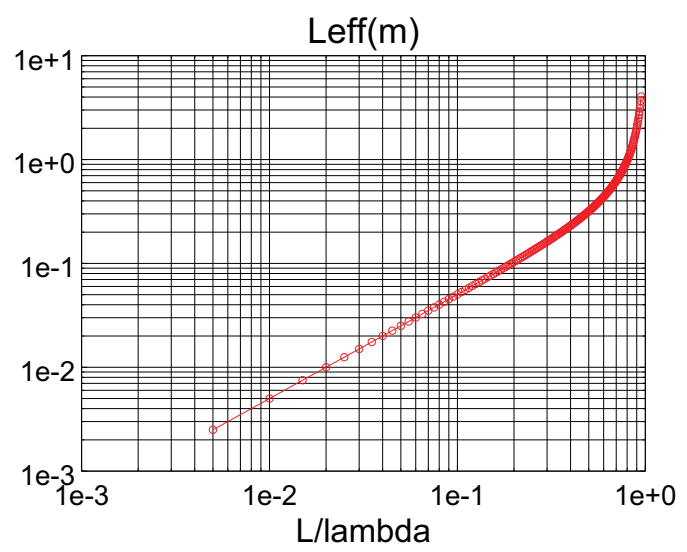

Figure 3. Effective length of the probe (short dipole): $L=0.48 \lambda(30 \mathrm{~mm})$. 


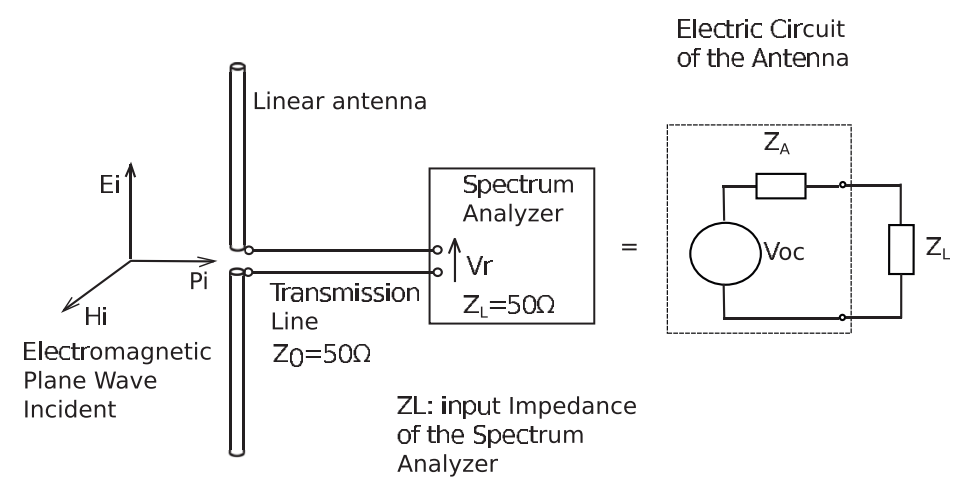

Figure 4. The receiving antenna with spectrum analyzer connected, and the incident electromagnetic plane wave, has the electric field parallel to the antenna.

The open circuit voltage can be expressed as:

$$
\left|V_{o c}\right|=\left|V_{r}\right|\left(\frac{\left|Z_{A}+Z_{L}\right|}{\left|Z_{L}\right|}\right)
$$

where $Z_{L}=50 \Omega$

From Eqs. (12) and (13):

$$
\left|V_{o c}\right|=\sqrt{W_{r}\left|Z_{L}\right|}\left(\frac{\left|Z_{A}+Z_{L}\right|}{\left|Z_{L}\right|}\right)
$$

Using the Eq. (11), the electric field incident to the probe is:

$$
\left|E_{i}\right|=\frac{\left|V_{o c}\right|}{L_{e f f}}
$$

From Eqs. (10), (14), and (15), the electric field incident to the dipole can, therefore, be obtained as:

$$
\left|E_{i}\right|=\frac{\left|V_{o c}\right|}{L_{e f f}}=\sqrt{\frac{W_{r}\left|Z_{L}\right| Z_{00} \cos \phi}{A_{e f f}}}
$$

where $Z_{L}=50 \Omega$ is the impedance of the spectrum analyzer; $Z_{A}$ is the input impedance of the antenna; $Z_{00}=120 \pi \Omega$ is the intrinsic impedance of the vacuum; $\phi$ is the phase of the impedance $Z_{L} ; A_{\text {eff }}$ is the effective area of the receiving antenna; and $W_{r}(W)$ is the power measured by the spectrum analyzer.

The natural parameter of a receiving antenna is the capture area, or effective area, and the natural parameter of a transmitting antenna is the gain [3]. Other parameters such as the transmitting antenna area, or the receiving antenna gain, are obtained by means of the natural parameters and the effective area of the isotropic radiator. 


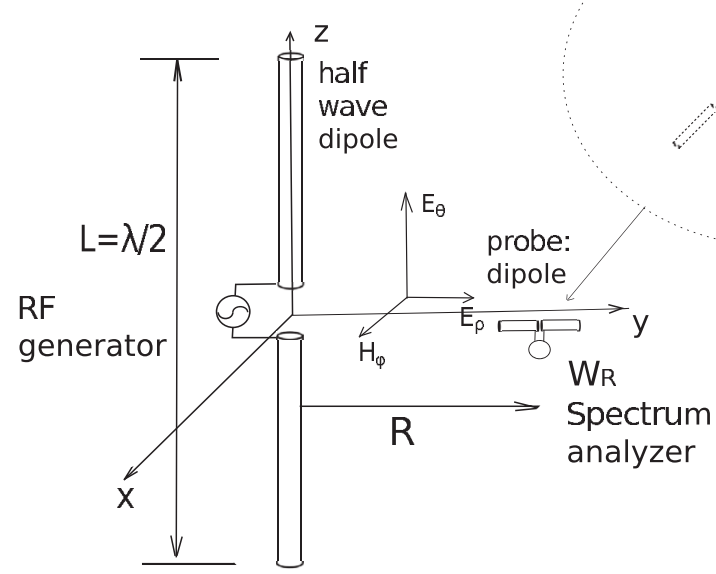

Figure 5. Measurement setup for $\mathrm{E}$ field components $E_{x}, E_{y}$, and $E_{z}$.

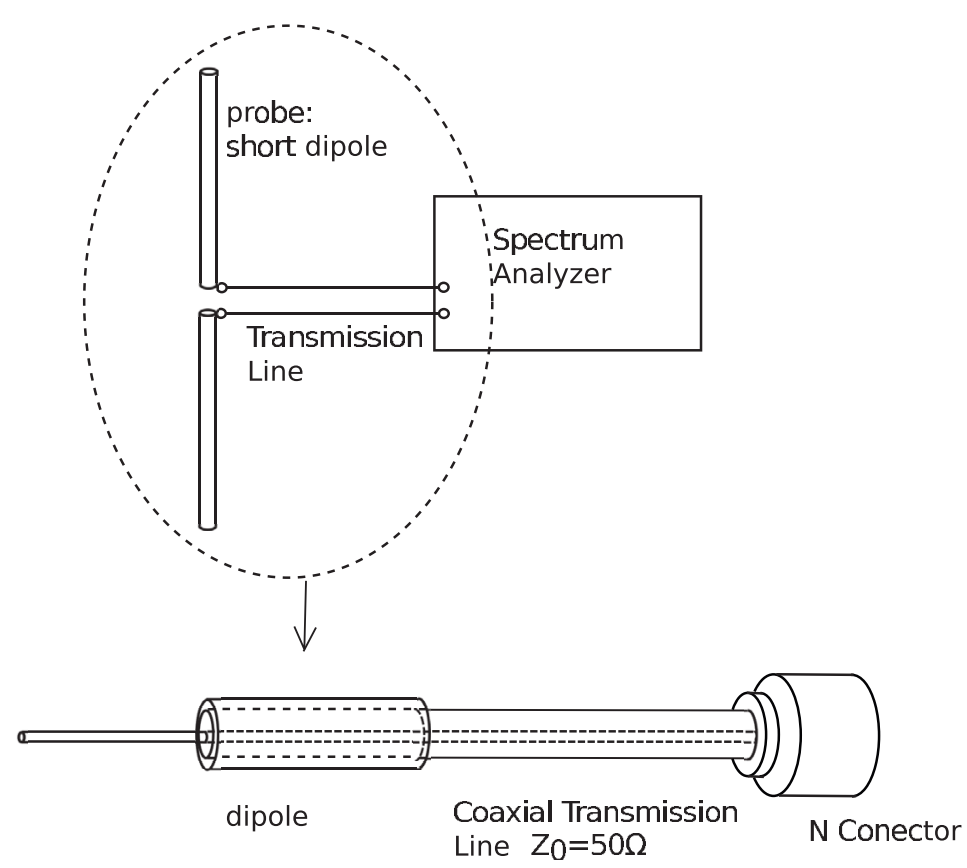

Figure 6. Simple electric field probe circuit. 


\subsection{Example of a radiating field from a dipole}

An example of the electric field measurement using a dipole probe is shown in Figure 5. A half wave dipole is radiating energy, and the probe (dipole) measures the induced voltage for the three axis: $E_{\theta}, E_{\rho}, E_{\phi}$. Figure 6 shows the electric field probe, composed of a dipole antenna, a coaxial transmission line, and the connections. This probe is connected to the spectrum analyzer in order to obtain the received voltage.

Figure 7a shows the received power measured with a dipole probe and Figure $7 \mathbf{b}$ shows the electric field calculated by means of Eq. (16). To obtain the electric field, it is necessary to know the effective length of the electric field probe $\left(L_{\text {eff }}\right)$, the impedance of the electric field probe $\left(Z_{A}\right)$, and the impedance of the spectrum analyzer $\left(Z_{L}\right)$.

Figure $8 \mathbf{a}$ and $\mathbf{8 b}$ shows the curve of the electric field component $E_{\rho}$ and $E_{\phi}$ as a function of distance.

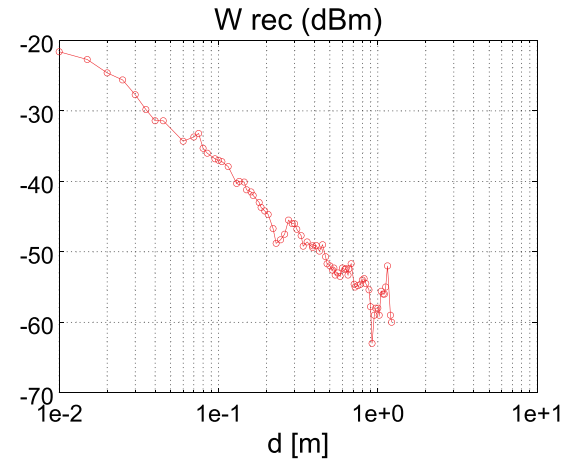

(a)

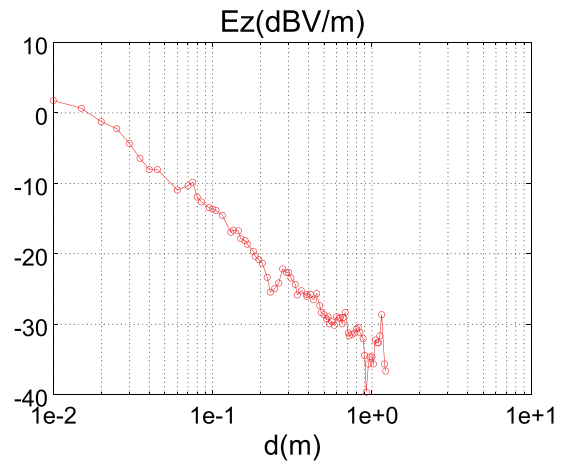

(b)

Figure 7. (a) Power measurement at the probe antenna terminals and (b) measured electric field component $E_{z}$.

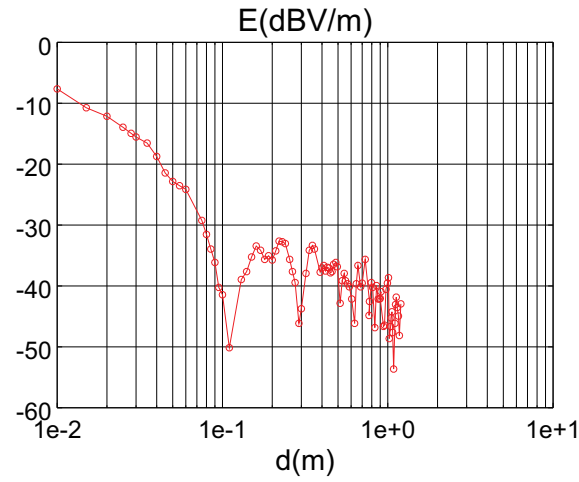

(a)

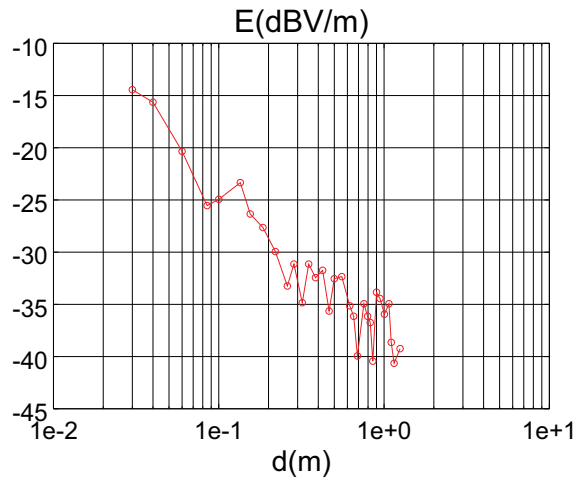

(b)

Figure 8. (a) Measured electric field component $E_{\rho}$ and (b) measured electric field component $E_{\phi}$. 


\section{Magnetic field probes}

A very useful method for measuring the magnetic field of electromagnetic waves is by means of a loop antenna, which can be used with one turn or several turns, where a radiating field is received. A simple loop antenna is shown in Figure 9a [1] and a shielded loop antenna is shown in Figure 9b [4].

In order to obtain the induced voltage at the terminals of the loop antenna, consider a short loop antenna, where the wavelength of the electromagnetic wave received is much greater than the perimeter of the one-turn antenna. The Maxwell-Faraday Law expression in differential form is [1]:

$$
\nabla \times \vec{E}=-\frac{\partial \vec{B}}{\partial t}
$$

where $B[T]$ is the magnetic flow density, $E[V / m]$ is the electric field, and $t[s]$ is the time.

If the electromagnetic wave has a harmonic variation as a function of the time, the electric and magnetic field can be expressed as [5]:

$$
\begin{aligned}
& E=E_{0} e^{j \omega t} \\
& H=H_{0} e^{j \omega t}
\end{aligned}
$$

From Eq. (17), making the integration at both sides:

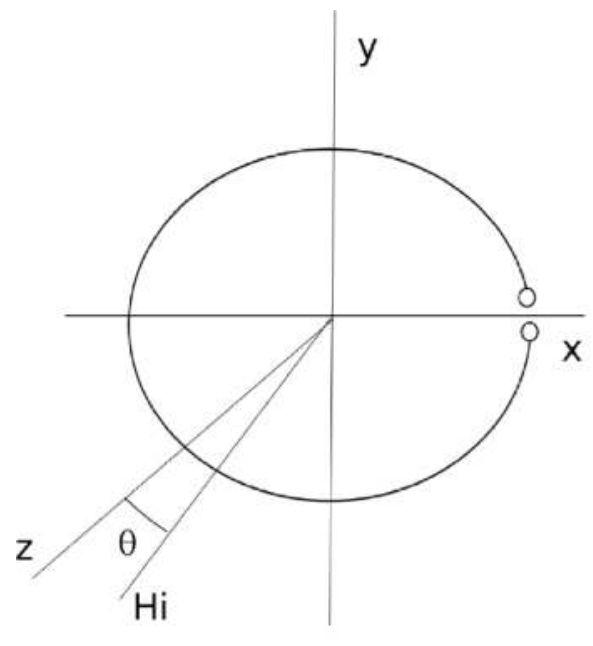

(a)

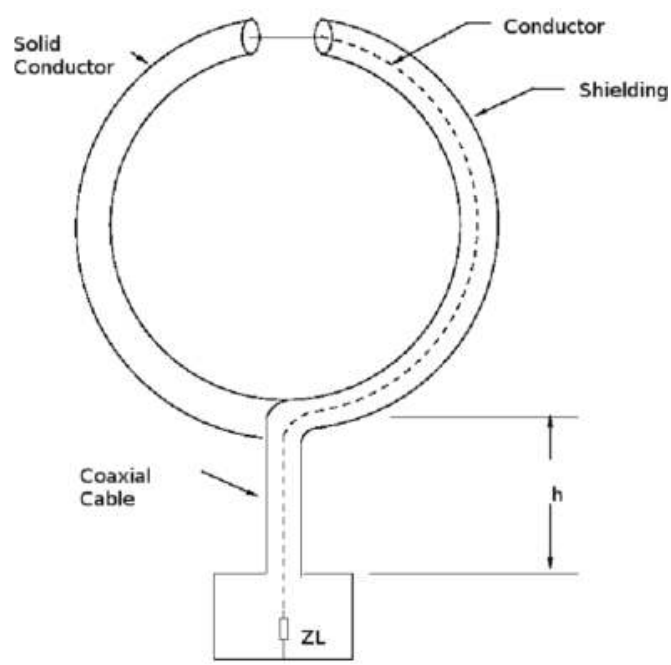

(b)

Figure 9. (a) Ideal Loop. (b) Shielding loop antenna. 


$$
\int_{s}(\nabla \times \vec{E}) \cdot \overrightarrow{d s}=-\int_{s} \frac{\partial \vec{B}}{\partial t} \cdot \overrightarrow{d s}
$$

From Eq. (20), applying the Stokes theorem, the electromotive force (FEM) is [1]:

$$
\text { fem }_{\text {induced }}=-\frac{\partial}{\partial t} \int_{s} \vec{B} \cdot \overrightarrow{d s}
$$

The loop antennas can be classified (into two classes) electrically small and electrically large antennas. The first is useful for the measurement of the magnetic field induced by the radiated electromagnetic field [4]. The electrically large loop antennas are those whose circumference is about a free-space wavelength $(C \cong \lambda)$. In this chapter, the electrically small loop antennas are discussed. The electrically small loop antennas are those whose overall length (number of turns by the length of the circumference) is usually less than one-tenth of a wavelength. This can be written as [2]:

$$
n \cdot C<\lambda / 10
$$

where $\mathrm{n}$ is the number of turns, $C[m]$ is the perimeter of the circumference of the loop, and $\lambda[m]$ is the wavelength.

In the small loop antennas, the current in the conductor of the antenna can be considered constant, i.e., $I(x, y, z)=I_{0}[2,4]$.

\subsection{Example}

A small loop antenna of radius $=10 \mathrm{~cm}$, with 50 turns, which bandwidth of interest is $200 \mathrm{kHz}$, then the number of turns by the perimeter of the circumference is

$$
n \cdot C=31.4 \mathrm{~m}
$$

The wavelength for the highest frequency is

$$
\lambda=\frac{c}{f}=\frac{3 \cdot 10^{8} \mathrm{~m} / \mathrm{s}}{200 \cdot 10^{3}}=1500 \mathrm{~m}
$$

Therefore,

$$
\frac{\lambda}{10}=150 \mathrm{~m}
$$

The condition of Eq. (22) is

$$
31.4<150 \mathrm{~m}
$$

Then, the condition of Eq. (22) has been satisfied for electrically small loops of a bandwidth of $200 \mathrm{kHz}[2,4]$. 
The magnetic field intensity inside the loop antenna shown in Figure $\mathbf{1 5}$ can be considered constant, then, Eq. (21) can be expressed as:

$$
\text { fem }_{\text {induced }}=-j \omega B \cos \theta A
$$

where $\omega=2 \pi f[\mathrm{rad} / \mathrm{s}], f[\mathrm{~Hz}]$ is the frequency, $B\left[\mathrm{~Wb} / \mathrm{m}^{2}\right]$ is the magnetic flux density, $A\left[\mathrm{~m}^{2}\right]$ is the area of the loop, and $\theta[\mathrm{rad}]$ is the angle between $\mathrm{z} y \mathrm{H}$.

As the constitutive relation between the incident magnetic field to the antenna $H$ and the magnetic flux density $B[6]$ :

$$
B=\mu_{0} H
$$

where $\mu_{0}$ is the magnetic permeability of the vacuum. Then,

$$
\text { fem }_{\text {induced }}=-j \omega \mu_{0} H \cos \theta A
$$

For the loop antenna of $\mathrm{n}$ turns, the induced FEM can be expressed as:

$$
\text { fem }_{\text {induced }}=-j n \omega \mu_{0} H \cos \theta A
$$

The amplitude of the induced FEM can be named open circuit voltage of the loop antenna $\left(V_{o c}\right)$ :

$$
V_{o c}=n \omega \mu_{0} H \cos \theta A
$$

If the loop antenna has a ferrite core, the magnetic permeability of the ferrite is $\mu=\mu_{o} \mu_{r^{\prime}}$ where $\mu_{r}$ is the relative magnetic permeability of antenna loop with a ferrite core, and the magnetic flux density is $B=\mu_{r} \mu_{0} H$.

\subsection{Electric circuit of the loop antenna}

The electric circuit of a loop antenna of $\mathrm{n}$ turns in receiving mode can be expressed by impedance $Z_{A}=R+j X$ in series with the ideal voltage generator. The spectrum analyzer can be represented by means of an impedance $Z_{L}=50 \Omega$. This equivalent Thevenin electric circuit is shown in Figure 18, where Voc is the open circuit voltage, $Z_{L}[\Omega]$ is the impedance of the spectrum analyzer, and $Z_{A}[\Omega]$ is the impedance of the loop antenna.

The measured voltage $V_{m}$ by the spectrum analyzer can be written as:

$$
V_{o c} \frac{Z_{L}}{Z_{L}+Z_{A}}=V_{m}
$$

From Eqs. (31) and (32), the magnetic field can be expressed like a function of the measured voltage $V_{m}$ : 


$$
H=V_{m} \frac{Z_{L}+Z_{A}}{Z_{L}} \frac{1}{n \omega A \mu_{0} \cos \theta}
$$

Therefore, the magnetic flux density results in (Figure 10):

$$
B=\mu_{0} H=V_{m} \frac{Z_{L}+Z_{A}}{Z_{L}} \frac{1}{n \omega A \cos \theta}
$$

\subsection{Antenna factor}

The definition of the antenna factor $K_{i}$, relates the magnetic field $H$, of the electromagnetic wave incident to the loop antenna with the current flowing in the loop $I_{m}$ [7]:

$$
K_{i}=\frac{H}{I_{m}}
$$

where

$$
I_{m}=\frac{V_{m}}{Z_{L}}
$$

From Eqs. (35) and (36), the antenna factor is

$$
K_{i}=\left(Z_{L}+Z_{A}\right) \frac{1}{n \omega A \mu_{0} \cos \theta}
$$

The antenna factor is important because it relates the current measured in the loop antenna and the incident unknown magnetic field.

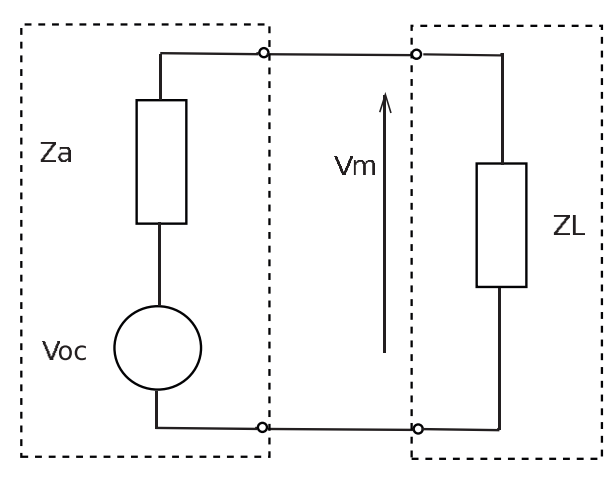

Antenna

Spectrum Analyzer

Figure 10. Electric circuit model of the loop antenna used for the measurement. 
Another way to define the antenna factor is:

$$
K_{v}=\frac{H}{V_{o c}}
$$

where the subindex shows if it is defined by the voltage (v) or current (i).

Using Eq. (31) $K_{v}$ results:

$$
K_{v}=\frac{1}{n \omega A \mu_{0} \cos \theta}
$$

Note that the relation between $K_{i}$ and $K_{v}$ is

$$
\frac{K_{i}}{K_{v}}=Z_{L}+Z_{A}
$$

\subsubsection{Example}

The antenna factor $K v$ of three short loop antennas has been computed by means of Eq. (39), as shown in Figures 11, 12, and 13. The antenna factor $K_{v}$ has a slope of $-20 \mathrm{~dB} / \mathrm{dec}$ and it is decreasing with $n, \omega, \mu$, and $A$. This behavior can be observed in Table 1.

Typically, the small loop antennas are used to measure the magnetic fields in a range of frequencies of $20 \mathrm{~Hz}-30 \mathrm{MHz}$ [8]. The loop antenna can also be used to measure the magnetic field strength emission from a device [9].

From the equation of the open circuit voltage of Eq. (31), $V_{o c}$ increases with the area and the number of turns $\mathrm{n}$. Both parameters can be used to select the voltage of reception and the sensibility of the measurement. The section of the wire selected has been chosen to reduce the losses as Joule effect.

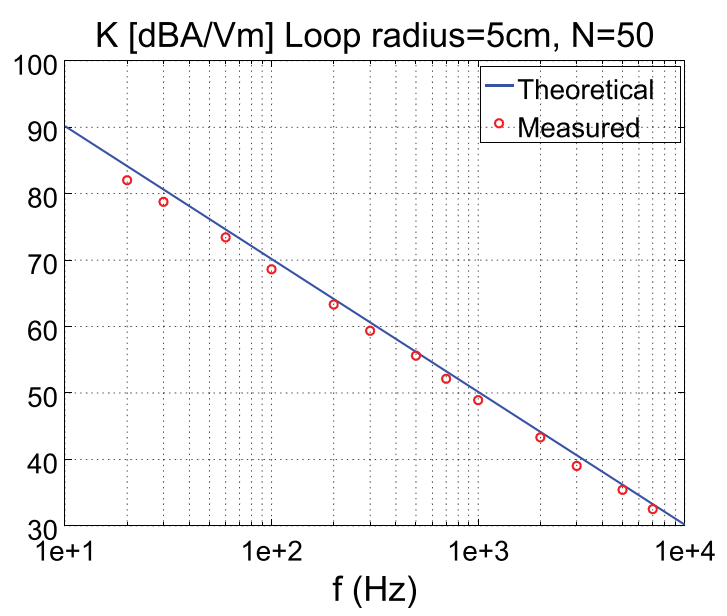

Figure 11. Antenna factor $K_{v}$ measured and calculated. Loop: radius $=0.05 \mathrm{~m}$ and $\mathrm{N}=50$. 


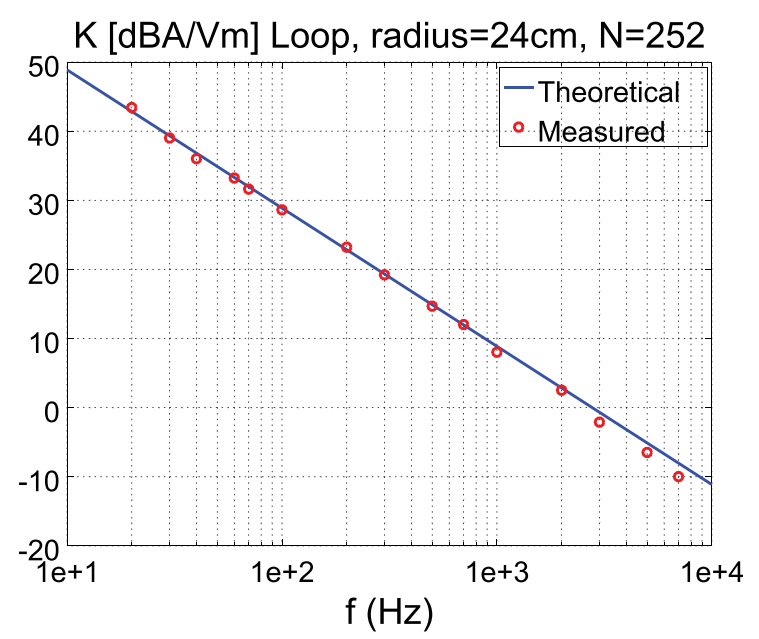

Figure 12. Antenna factor $K_{v}$ measured and calculated. Loop: radius $=0.24 \mathrm{~m}$ and $\mathrm{N}=252$.

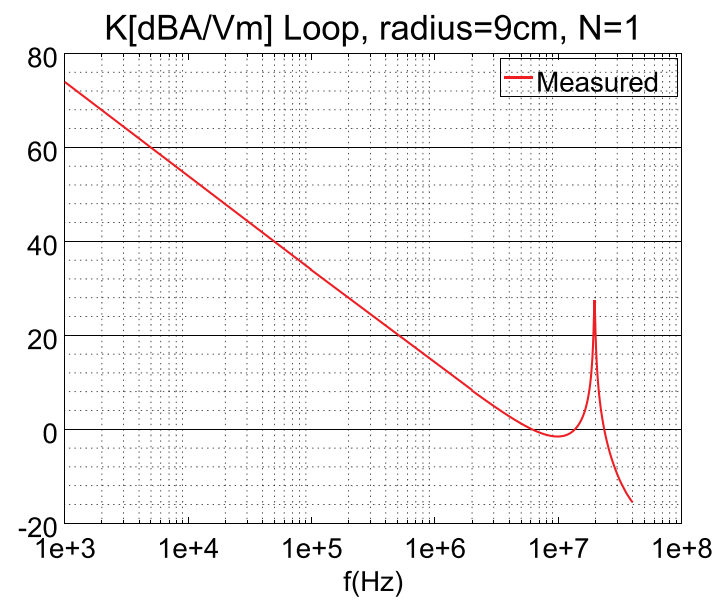

Figure 13. Antenna factor $K_{v}$ measured. Loop: radius $=0.09 \mathrm{~m}$ and $\mathrm{N}=1$.

\begin{tabular}{lll}
\hline$a$ & $\mathrm{n}$ & $\boldsymbol{K}_{v}\left(\mathrm{dBA} / \mathrm{V}_{\mathbf{m}}\right) \mathbf{f}=\mathbf{1} \mathbf{k H z}$ \\
\hline 0.05 & 50 & 50 \\
0.24 & 252 & 8 \\
0.09 & 1 & 75 \\
\hline
\end{tabular}

Table 1. Antenna factors for three different loop antennas. 
Currently, the measurement of magnetic field emitted by electronic devices is done to verify the electromagnetic compatibility [9, 10] or the emissions in the nature [11]. In the magnetic field measurement, a calibrated measurement system is required, where the probes are excited by the uniform magnetic field [12] to obtain the antenna factor [13]. The magnetic field to be measured should be in a near-field zone [14] or in a far-field zone [5].

\section{Example}

A measurement of the power supply of a personal computer is shown in Figure 14a and 14b. The hotspots are indicated with dark grey where the emission is highest. The magnetic field probe made with a ferrite core, which has been used in this measurement, is shown in Figure 15.
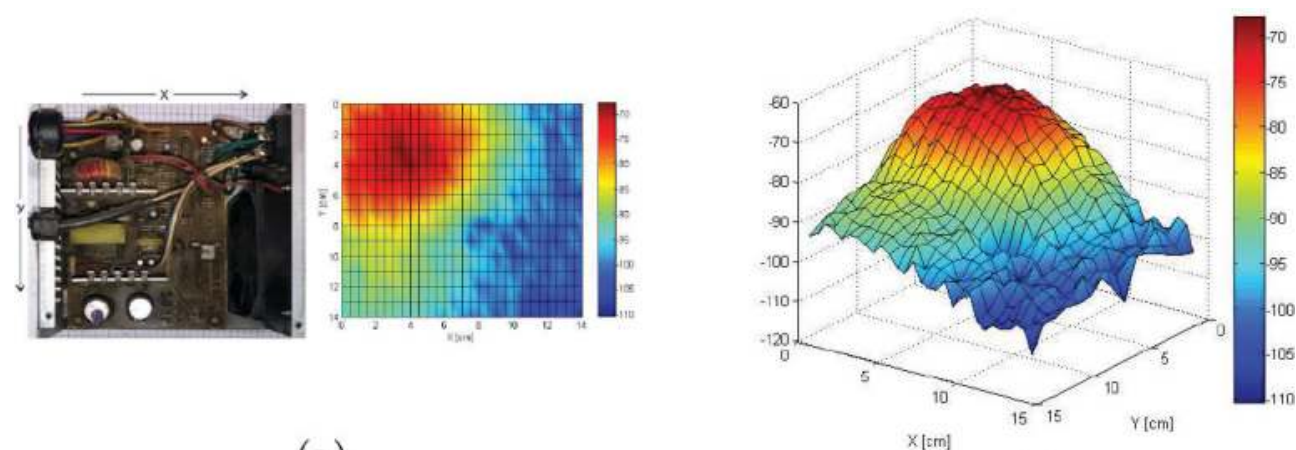

(a)

(b)

Figure 14. (a) Power supply in a 2D picture of emission. (b) Emission of the power supply versus $x, y$.

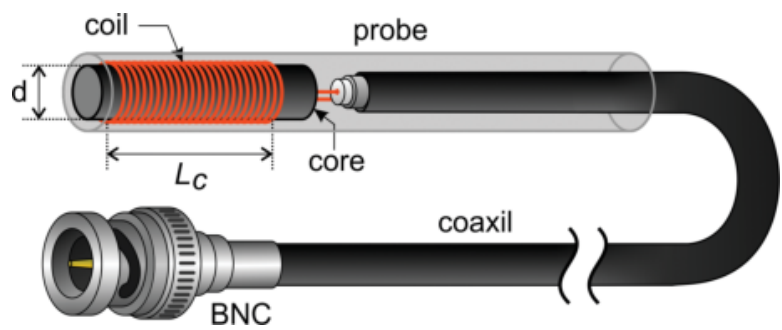

Figure 15. The magnetic field probe with a coil built with ferrite core.

\section{Helmholtz coils}

The Helmholtz coils are the most simple configuration to produce a magnetic field relatively constant. Helmholtz coils are two circular coils coaxial with the same radius, which is equal to the distance between the planes of the coils [15]. When the current flows in opposite directions, the configuration is called anti-Helmholtz coils. The cancellation of the magnetic field is important in various applications such as measurements, bioengineering investigations, and 
calibrations of probes and sensors. In Helmholtz coils expand the area of uniformity of the magnetic field, and reduce the variation of the field in the axes it is interesting. Based on the study of a two-coil system, it has been determined that three coils through which current flows produce magnetic field with better characteristics in intensity and uniformity than the standard Helmholtz coils. The polygonal coils are easier to be produced in the industry. [16]. When the number of sides of a polygon forming the coil is increased, the distribution on the intensity of magnetic field is more uniform [16, 17]. If greater uniformity of the magnetic field is required, a five-rectangular coils system for measuring biomagnetics can be used [16, 18]. Eight Helmholtz coils are used to calibrate a magnetometer in a uniform magnetic field system [19] (Figures 16 and 17).

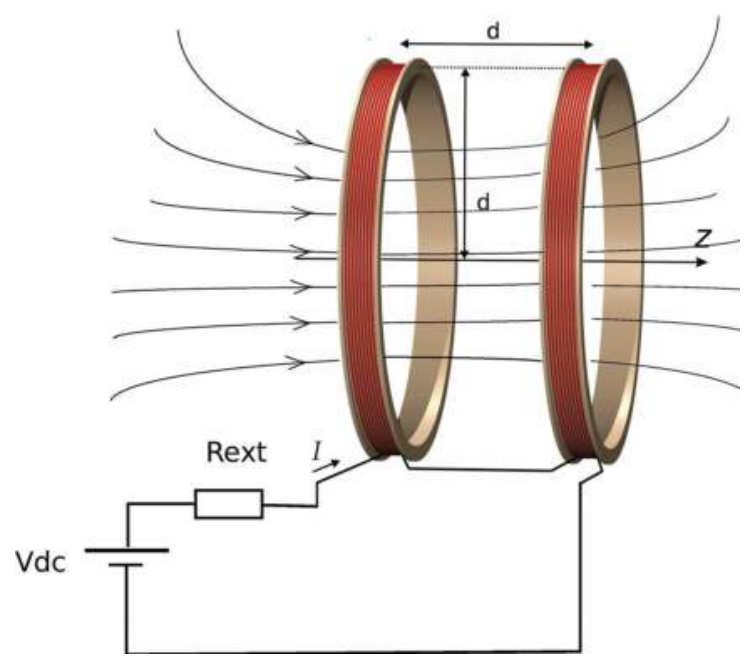

Figure 16. Picture of the Helmholtz coils.

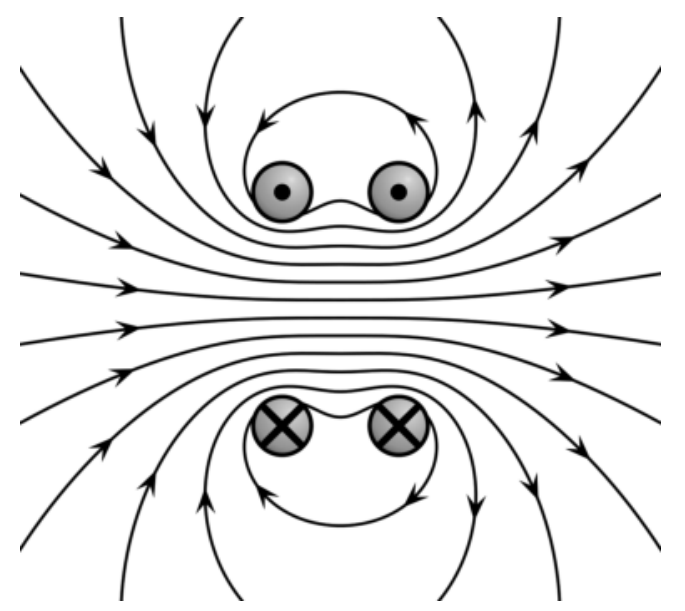

Figure 17. Magnetic field lines of the Helmholtz coils. 


\subsection{Helmholtz coils with DC current applied}

In the magnetic flux density vector $\vec{B}$, produced by two coils, as shown in Figure 16, where a DC current I is flowing by the coils, two identical terms are obtained. The coils are placed at $\mathrm{z}=-\mathrm{d} / 2$ and $\mathrm{z}=+\mathrm{d} / 2$.

The magnetic flux density $\vec{B}$ of the Helmholtz coils with the radius equal to the distance between coils has first and second derivatives of zero at $z=0 \frac{\partial B}{\partial z}=\frac{\partial^{2} B}{\partial z^{2}}=0[20]$. Then, the magnetic flux density is uniform at $z=0$ :

$$
\left.\overrightarrow{B(r)}\right|_{z=0}=\frac{\mu_{0} I}{\left(\frac{5}{4}\right)^{3 / 2} a} \widehat{z}
$$

The magnetic flux density can be expanded by Taylor series, and then the deviation from $\left.\overrightarrow{B(z)}\right|_{z=0}$ is $B(z)=B(0)\left(1 \pm 1.510^{-4}\right)$ at $|z|<\frac{a}{10}$.

\subsection{Helmholtz coils with AC current applied computed in any point in the space}

Consider two coils connected to an AC generator, where a sinusoidal current $I(t)$ flows as shown in Figure 18. The Helmholtz coils are used in low frequencies: LF, VLF, and ULF. For these frequencies, the wavelength is much larger than the dimensions of the loop. A number of turns is used in each winding that make up the Helmholtz coils, where the number of turns of the coil (n), multiplied by the perimeter of the circumference (C), is much less than a wavelength $N C<\lambda$, which can be enunciated as [2]:

$$
N C<\frac{\lambda}{10}
$$

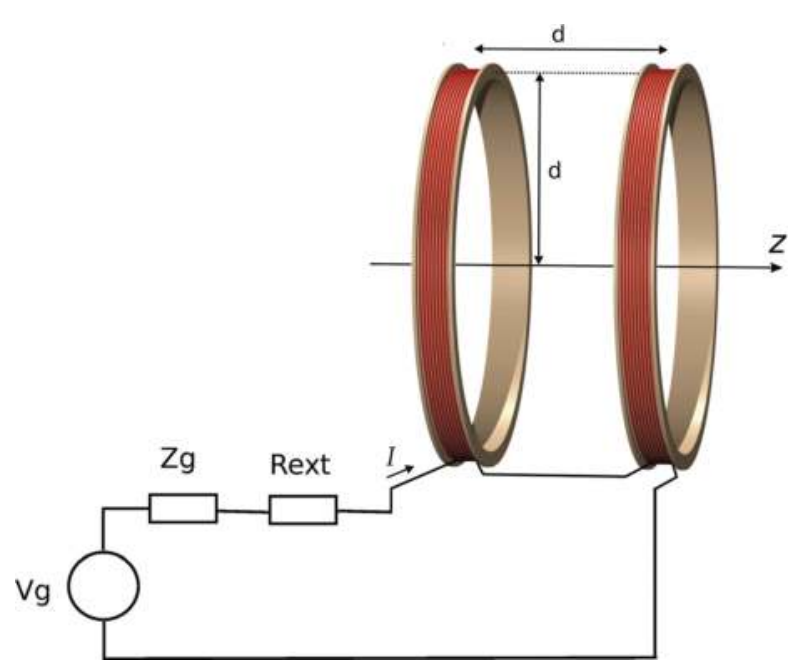

Figure 18. AC current connected to the Helmholtz coils. 
To the frequencies that fulfill the relation (42), the current is practically constant in each point of the Helmholtz coils, and it will only be variable with the time. The vectorial $\vec{A}$ can be written as [2]:

$$
\vec{A}=\frac{\mu_{0}}{4 \pi} \int_{v} \frac{\overrightarrow{J(r)} d v^{\prime}}{R}
$$

For the particular case of the coil can be considered a linear wire where the current flows, the expression (43) is

$$
\vec{A}=\frac{\mu_{0}}{4 \pi} \oint_{c} I \frac{\overrightarrow{d l^{\prime}}}{R}
$$

where $\overrightarrow{d l^{\prime}}=\left(-a \operatorname{sen} \phi^{\prime}, a \cos \phi^{\prime}, 0\right) d \phi^{\prime}$

$\vec{r}=(r \operatorname{sen} \theta, 0, r \cos \theta)$

$r^{\prime}=\left(a \cos \phi^{\prime}, a \operatorname{sen} \phi^{\prime}, 0\right)$

$R=\left|\vec{r}-\overrightarrow{r^{\prime}}\right|=\sqrt{r^{2}+a^{2}-2 \operatorname{rasen} \theta \cos \phi^{\prime}}$

The current intensity I is constant at the perimeter of the loop [2], then, the vector $\widetilde{A}$ results in:

$$
\vec{A}=\frac{\mu_{0}}{4 \pi} \oint_{0} \pi \frac{\widehat{\phi} I \cos \phi^{\prime} d \phi^{\prime}}{\sqrt{r^{2}+a^{2}-2 r a s e n \theta \cos \phi^{\prime}}}
$$

that is, the Fredholm integral of the first kind [21], this results in:

$$
\vec{A}=\frac{\mu_{0} I}{\pi k} \sqrt{\frac{a}{\rho}} \cdot\left[\left(1-\frac{1}{2} k^{2}\right) \cdot K\left(k^{2}\right)-E\left(k^{2}\right)\right]
$$

where $\rho=\sqrt{r^{2}-z^{2}}, k^{2}=\frac{4 a \rho}{(a+\rho)^{2}+z^{2}}, K(k)=\int_{0}^{\pi / 2} \frac{d \phi}{\sqrt{1-k^{2} \operatorname{sen}^{2} \phi}}$ eliptic integral of the first kind. $E(k)=\int_{0}^{\pi / 2} \sqrt{1-k^{2} \operatorname{sen}^{2} \phi} d \phi$ eliptic integral of the second kind.

Then, $\vec{B}=\nabla \times \vec{A}$ can be computed [21]:

$$
\begin{aligned}
& \overrightarrow{B_{\rho}}=\frac{\mu_{0} I}{2 \pi} \frac{z}{\rho \sqrt{(\rho+a)^{2}+z^{2}}} \cdot\left[\frac{\rho^{2}+a^{2}+z^{2}}{(a-\rho)^{2}+z^{2}} \cdot E\left(k^{2}\right)-K\left(k^{2}\right)\right] \\
& \overrightarrow{B_{z}}=\frac{\mu_{0} I}{2 \pi} \frac{1}{\sqrt{(\rho+a)^{2}+z^{2}}} \cdot\left[\frac{a^{2}-\rho^{2}-z^{2}}{(a-\rho)^{2}+z^{2}} \cdot E\left(k^{2}\right)-K\left(k^{2}\right)\right]
\end{aligned}
$$

The total magnetic flux density of the Helmholtz coils, where the coils are placed at $z=-d / 2$ and $z=+d / 2$ is 


$$
\begin{aligned}
& B_{\rho}=B_{\rho}(\rho, z+d)+B_{\rho}(\rho, z-d) \\
& B_{z}=B_{z}(\rho, z+d)+B_{z}(\rho, z-d)
\end{aligned}
$$

\subsection{Example}

The Helmholtz coils have been built with two coils of radius $a=0.39 \mathrm{~m}$, number of turns $N=8$, space between coils $s=a=0.39 \mathrm{~m}$, with the AC current applied to the coils of $|I| \cong 15 \mathrm{~mA}$ at $f=1 \mathrm{kHz}$. The magnetic flux density can be calculated with Eq. (41), thus:

$$
\left.\vec{B}\right|_{z=0}=\frac{4 \pi 10^{-7} \mathrm{H} / \mathrm{m} 15 \cdot 10^{-3} A}{\left(\frac{5}{4}\right)^{3 / 2} 0.39 \mathrm{~m}}
$$

Results:

$$
\left.\vec{B}\right|_{z=0}=27.6 \mu T \widehat{z}
$$

In Figure 19a, the magnetic flux density as a function of $\mathrm{z}$ for $\rho=0$ has been plotted, and the relative error of the magnetic flux density as a function of $z$ and $\rho$ in a 3D plot is shown in Figure 19b.

In the zone where the magnetic flux density is uniform, at $z=0$, the probes to be calibrated are placed, at a low frequency signals from DC up to $200 \mathrm{kHz}$, the Helmholtz coils are used. The errors of the magnetic flux density B can be calculated using Eqs. (47), (48), (49) and (50).

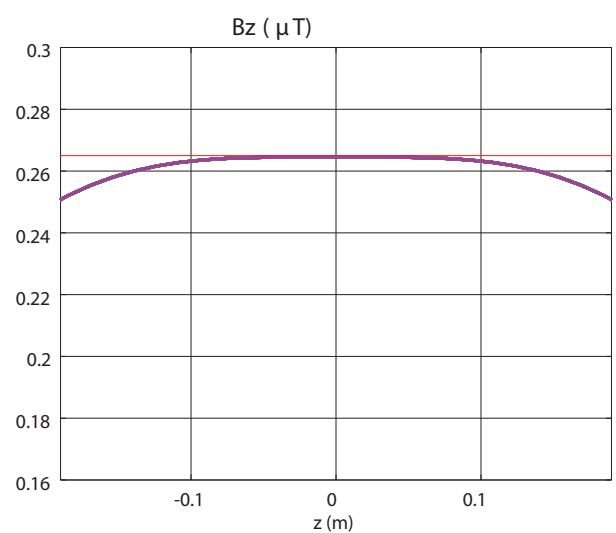

(a)

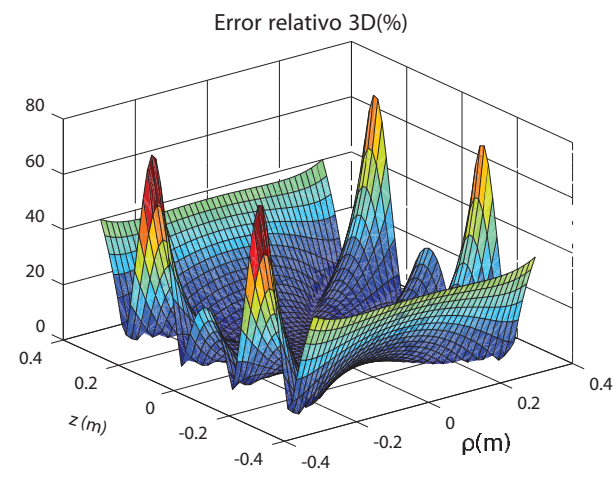

(b)

Figure 19. (a) Magnetic flux density $B z(z)$ at $\rho=0$ and (b) relative error of the magnetic flux density $B$ with respect to $B(z=0)$. 


\section{Author details}

Walter Gustavo Fano

Address all correspondence to: gustavo.gf2005@gmail.com

Faculty of Engineering, Universidad de Buenos Aires, Buenos Aires, Argentina

\section{References}

[1] Trainotti V, Fano WG. Ingenieria Electromagnetica. Vol. 1, 1st ed. Buenos Aires: Nueva Libreria; 2004. p. 512

[2] Balanis CA. Antenna Theory: Analysis and Design. Hoboken: Wiley-Interscience; 2005

[3] Trainotti V. Electromagnetic compatibility (emc) antenna gain and factor. IEEE Transactions on Electromagnetic Compatibility. 2017;59:1006-1015

[4] Johnson R, Jasik H. Antenna Engineering Handbook, Electronics: Electrical engineering. New York: McGraw-Hill; 1993

[5] Trainotti V, Fano WG, Dorado L. Ingenieria Electromagnetica. Vol. 2, 1st ed. Buenos Aires: Nueva Libreria; 2005. p. 1007

[6] Van Bladel J. Electromagnetic Fields. Hoboken: Wiley; 2007

[7] Osburn JDM. EMC antenna parameters and their relationships. Interference Technology; 2012. https://interferencetechnology.com/emc-antenna-parameters-and-their-relationships/\#

[8] Montrose MA, Nakauchi EM. Test for EMC Compliance, Approach and Techniques. Hoboken: Wiley; 2004

[9] Fano WG. RF Emissions of compact fluorescent lights. Interference Technology. Vol. Europe EMC Guide; 2013. https://interferencetechnology.com/rf-emissions-of-compact-fluorescentlights/

[10] Paul C. Introduction to Electromagnetic Compatibility, Wiley Series in Microwave and Optical Engineering. Hoboken: Wiley; 2006

[11] Maffia E, Trainotti V, Fano WG, Trench N. Medición de la resonancia de schumann. Latinmag Letter. 2011;1(A02):1-8

[12] Pahl RA, Rovey JL, Pommerenke DJ. Calibration of magnetic field probes at relevant magnitudes. In: Proceedings of the 2013 19th IEEE Pulsed Power Conference (PPC). 1621 June 2013. San Francisco, CA, USA: IEEE; 2013. pp. 1-6

[13] Fano WG, Alonso R, Carducci LM. Near field magnetic probe applied to switching power supply. In: Proceedings of the 2016 IEEE Global Electromagnetic Compatibility Conference (GEMCCON). 7-9 Nov 2016. Mar del Plata, Argentina: IEEE; 2016. pp. 1-4 
[14] Trzaska H. Electromagnetic Field Measurements in the Near Field. Atlanta: Noble Publishing; 2001

[15] Petrovik DM, Radic MD. Generalization of Helmholtz coil problem. Serbian Journal of Electrical Engineering. 2015;12:375-384

[16] Merritt R, Purcell C, Stroink G. Uniform magnetic field produced by three, four, and five square coils. Review of Scientific Instruments. 1983;54(7):879-882

[17] Lu X, Tian J. Simulation for the polygonal coil magnetic field. Simulation for the Polygonal Coil Magnetic Field. 2013;6:43-49

[18] Wang J, She S, Zhang S. An improved Helmholtz coil and analysis of its magnetic field homogeneity. Review of Scientific Instruments. 2002;73(5):2175-2179

[19] Baranov P, Baranova V, Uchaikin S, Pisarenko Y. Creating a uniform magnetic field using axial coils system for calibration of magnetometers. In: Proceedings of the 2016 Dynamics of systems, Mechanisms and Machines (Dynamics). 15-17 Nov. 2016. Omsk, Russia. pp. 1-5

[20] Fernandez JC. Ingenieria Electromagnetica. I Modelos estáticos y circuitales. Vol. 1, 1st ed. Buenos Aires: Eudeba; 2013. p. 690

[21] Dolezel I, Pavel K, Pavel S. Integral Methods in Low-Frequency Electromagnetics. Hoboken: Wiley; 2009 